Recepción: 20 / 01 / 2019

Aceptación: 19 / 03 / 2019

(c) $(1)(90$

Ciencias de la salud

Publicación: 05 / 05 / 2019

Artículo de Revisión

\title{
Mecanismos de prevencion para reducir problemas con el oido causado por la exposición al ruido
}
Prevention mechanisms to reduce problems with the ear caused by exposure to noise

\section{Mecanismos de prevenção para reduzir problemas com o ouvido causados pela exposição ao ruído}

\author{
Marco V. Peñaherrera-Alcivar ${ }^{\mathrm{I}}$
}

dr.marco.palcivar@hotmail.com

Marcos A. Bedoya-Romo II

marcos4786@hotmail.es

Pierina E. Saltos-Montes III

pierina_s.m@hotmail.com

Evelyn E. Calderón-López IV

ivitef89@gmail.com

Marvin D. Veliz-Mero v

drmarveliz@hotmail.com

Marcos A. Campozano-Burgos VI

andres_bigman25@hotmail.com

Correspondencia: dr.marco.palcivar@hotmail.com

I. Médico General; Maestrante de Seguridad y Salud Laboral; Médico Ocupacional del distrito 13D02; Manta, Ecuador.

II. Médico Cirujano; Médico Residente de UCI del Hospital General IESS; Manta, Ecuador.

III. Médico Cirujano; Médico Residente de Emergencia del Hospital General IESS; Manta, Ecuador.

IV. Médico Cirujano; Médico Residente de Neonatología del Hospital General IESS; Manta, Ecuador.

v. Médico Cirujano; Médico Residente de Cirugía del Hospital General IESS; Manta, Ecuador.

VI. Médico Cirujano; Médico Residente de UCI del Hospital General IESS; Manta, Ecuador. 


\title{
Resumen
}

La importancia de cuidar la audición depende de cada individuo, el siguiente trabajo de investigación estudia los mecanismos de prevención que se deben considerar para reducir los problemas causados en el oído por la exposición al ruido, a través de una revisión bibliográfica que se enfoca en temas relacionados al de interés y que brindaron aportes para la investigación. El trabajo hace mención al oído como uno de los órganos más importantes del ser humano y a la pérdida de audición que se presenta de acuerdo a ciertos ambientes laborales que producen ruidos peligrosos y que pueden generar lo que se le conoce como pérdida de audición inducida, considerando, que este tipo de problemas puede prevenirse si se toman las medidas de protección y si se mantienen los controles y estudios de investigación necesarios en las empresas con el fin de cuidar la audición de cada uno de sus trabajadores y evitar daños que luego podrían ser irreversibles. Durante la investigación se observa, cómo los trabajadores que están expuestos a ruidos peligros por años van perdiendo la capacidad de oír motivo por el cual, se hace mención a los Programas de Prevención Auditiva del Instituto de Seguridad y Salud Ocupacional (NIOSH) encargados de realizar las evaluaciones y controles adecuados con tal de garantizar la audición de sus empleados. Los mecanismos de prevención deben ser una prioridad para toda empresa y para todo individuo, está de parte del empleado cuidar de sí y utilizar los equipos de protección sin tener que recibir llamados de atención para su uso por parte del personal de seguridad de la empresa, así como también, la empresa debe velar por chequeo médico para todos aquellos que trabajen en ambientes de ruidos peligrosos.

Palabras claves: Oído; Audición; Pérdida de Audición; Prevención; Seguridad Ocupacional.

\begin{abstract}
The importance of caring for hearing depends on each individual, the following research work studies the prevention mechanisms that should be considered to reduce the problems caused in the ear by exposure to noise, through a bibliographic review that focuses on issues related to the interest and that provided contributions for the investigation. The work makes mention of the ear as one of the most important organs of the human being and the loss of hearing that occurs according to certain work environments that produce dangerous noises and that can generate what is known as induced hearing loss, considering, that this type of problem can be prevented if protection measures are taken and if the controls and research studies necessary in the companies are maintained in order
\end{abstract}


Mecanismos de prevencion para reducir problemas con el oido causado por la exposición al ruido

to take care of the hearing of each of their workers and avoid damages that could later be irreversible . During the investigation it is observed, how workers who are exposed to noise hazards for years are losing the ability to hear why, mention is made of the Auditory Prevention Programs of the Institute of Occupational Safety and Health (NIOSH) responsible for performing the appropriate evaluations and controls in order to guarantee the hearing of its employees. The prevention mechanisms should be a priority for every company and for every individual, it is for the employee to take care of himself and use the protective equipment without having to receive calls for attention for its use by the security personnel of the company, as well as, the company must ensure a medical checkup for all those who work in hazardous noise environments.

Keys words: Hearing; Hearing; Hearing Loss; Prevention; Occupational Safety.

\section{Resumo.}

A importância de cuidar da audição depende de cada indivíduo, o trabalho de pesquisa a seguir estuda os mecanismos de prevenção que devem ser considerados para reduzir os problemas causados ao ouvido pela exposição ao ruído, através de uma revisão bibliográfica que enfoca questões relacionadas ao interesse e que forneceu contribuições para a investigação. O trabalho faz menção ao ouvido como um dos órgãos mais importantes do ser humano e a perda auditiva que ocorre de acordo com determinados ambientes de trabalho que produzem ruídos perigosos e que podem gerar o que se conhece como perda auditiva induzida, considerando que este tipo de problema pode ser evitado se medidas de proteção forem tomadas e se os controles e estudos de pesquisa necessários nas empresas forem mantidos para cuidar da audiência de cada um de seus trabalhadores e evitar prejuízos que mais tarde poderiam ser irreversíveis. Durante a investigação observa-se como os trabalhadores que estão expostos a riscos de ruído há anos perdem a capacidade de ouvir o porquê, mencionam-se os Programas de Prevenção Auditiva do Instituto de Segurança e Saúde Ocupacional (NIOSH) responsáveis pela realização das avaliações apropriadas. e controles, a fim de garantir a audição de seus funcionários. Os mecanismos de prevenção devem ser uma prioridade para cada empresa e para cada indivíduo, é para o funcionário cuidar de si e usar o equipamento de proteção sem ter que receber chamadas de atenção para o seu uso pelo pessoal de segurança da empresa, bem como como, a empresa deve garantir um check-up médico para todos aqueles que trabalham em ambientes perigosos.

Palavras chaves: Audição; Audição; Perda de audição; Prevenção; Segurança no trabalho. 


\section{Introducción.}

El oído forma parte de los órganos del cuerpo humano que brindan una de las funciones principales y de los cinco sentidos de la persona, estos órganos son sumamente delicados y requiere de cuidado y protección. El oído está compuesto por tres partes que trabajan en simultáneo para poder detectar los sonidos y transmitirlos al cerebro: el oído externo, el oído medio y el oído interno.

Debido a la cercanía que existe entre el oído externo y el medio ambiente y la conexión que hay entre el oído medio y la nariz se tienen varios factores que pueden afectar sobre este órgano y ocasionar problemas auditivos.

Uno de ellos es la pérdida de audición inducida por el ruido que representa el problema número uno de las discapacidades ocupacionales. Esta afección por lo generalmente es indoloro pero progresivo en el tiempo y lo más triste es que es permanente e inevitable. La pérdida de audición relacionada con el trabajo sigue siendo uno de los temas más críticos de seguridad y salud laboral, el National Institute for Occupational Safety and Health (2008) o según sus siglas en ingles (NIOSH) expresa que la pérdida de audición representa uno de los problemas en áreas prioritarias de investigación de estos tiempos.

La pérdida de audición es totalmente prevenible si se toman las medidas o mecanismos necesarios para evitar este problema, sin embargo, una vez que se adquiere es permanente e irreversible, por tal motivo los trabajadores y empleados deben tomar medidas de protección que garantice la audición y la NO exposición excesiva al ruido quedando de parte del personal tomar conciencia en la utilización de los equipos de seguridad que proporcione la empresa para su protección.

Adicional a esto, diariamente estamos expuestos a ruidos en el medio ambiente que también podrían causar problemas en la audición, el National Instituteon Deafness and other Communication Disorders (2018) indica, que aquellos sonidos que vienen del televisor y la radio, los aparatos electrodomésticos y el tráfico, forman parte de los ruidos del día a día y a los que normalmente estamos expuesto, a pesar de que los oímos a niveles que no afectan nuestra audición, se debe considerar que si este tipo de sonidos son altos y recurrentes pueden ser dañinos, así duren poco o mucho tiempo. Estos ruidos pueden dañar las estructuras delicadas del oído interno, causando pérdida de audición inducida por el ruido. 
Mecanismos de prevencion para reducir problemas con el oido causado por la exposición al ruido

El autor (FREMAP, 2018) en su estudio acerca de de las Recomendaciones Básicas de Seguridad y Salud en la Exposición Laboral al Ruido expresa, que el oído humano detecta sonidos cuya frecuencia está comprendida entre 16 y $16000 \mathrm{~Hz}$, e intensidad de 0 a $140 \mathrm{~dB}$. La frecuencia conversacional está entre 250 y $4000 \mathrm{~Hz}$, y la palabra se emite a una intensidad entre 30 y $70 \mathrm{~dB}$. La percepción de las variaciones de la presión del aire por parte del oído es lo que se conoce como sonido, mientras que el ruido comprende todos aquellos sonidos que se caracterizan por ser desagradables y presentarse con una cierta intensidad.

El ruido cuando es a nivel industrial está representado por aquellos sonidos que cumplen ambas características (sonido y ruido) que se genera en todo proceso industrial, el cual debe ser controlado por parte de los trabajadores para minimizar los riesgos de problemas de audición a futuro.

Las empresas y organizaciones deben mantener un control adecuado de los ruidos y comprobar frecuentemente que las medidas de prevención aprobadas para controlar el ruido tengan un eficiente funcionamiento. De acuerdo a la exposición al ruido por parte de los trabajadores, el personal tiene derecho a una revisión sanitaria adecuada con el fin de mantener supervisado los riesgos de pérdida de audición.

Mediante la redacción de este trabajo investigativo se pretende dar a conocer los procesos de prevención que se deben llevar a cabo para reducir los problemas que se generan cuando se tiene una excesiva exposición al ruido, con la finalidad de crear conciencia en aquellas personas que se encuentran constantemente expuestas a sonidos fuertes y desagradable para disminuir los riesgo de la aparición de la pérdida de audición inducida.

\section{Métodos y materiales.}

Para el desarrollo de este proceso investigativo, se plantea como metodología la encaminada hacia una orientación científica particular que se encuentra determinada por la necesidad de indagar en forma precisa y coherente una situación, en tal sentido (Davila, 2015) define la metodología "como aquellos pasos previos que son seleccionados por el investigador para lograr resultados favorables que le ayuden a plantear nuevas ideas”. (p.66) 
Marco V. Peñaherrera-Alcivar; Marcos A. Bedoya-Romo; Pierina E. Saltos-Montes; Evelyn E. Calderón-López; Marvin D. Veliz-Mero; Marcos A. Campozano-Burgos

Lo citado por el autor, lleva a entender que el desarrollo de la labor investigativa busca simplemente coordinar acciones enmarcadas en una revisión bibliográfica con el fin de complementar ideas previas relacionados a los Mecanismos de prevención para reducir problemas con el oído causado por la exposición al ruido, a través de una revisión de literatura, para así finalmente elaborar un cuerpo de consideraciones generales que ayuden a ampliar el interés propuesto.

\section{Tipo de Investigación}

Dentro de toda práctica investigativa, se precisan acciones de carácter metodológico mediante las cuales, se logra conocer y proyectar los eventos posibles que la determinan, así como las características que hacen del acto científico un proceso interactivo ajustado a una realidad posible de ser interpretada. En este sentido, se puede decir, que la presente investigación corresponde al tipo documental, definido por Castro (2016), "se ocupa del estudio de problemas planteados a nivel teórico, la información requerida para abordarlos se encuentra básicamente en materiales impresos, audiovisuales y /o electrónicos". (p.41).

En consideración a esta definición, la orientación metodológica permitió la oportunidad de cumplir con una serie de actividades inherentes a la revisión y lectura de diversos documentos donde se encontraron ideas explicitas relacionadas con los tópicos encargados de identificar a cada característica insertada en el estudio. Por lo tanto, se realizaron continuas interpretaciones con el claro propósito de revisar aquellas apreciaciones o investigaciones propuestas por diferentes investigadores relacionadas con el tema de interés, para luego dar la respectiva argumentación a los planteamientos, en función a las necesidades encontradas en la indagación.

\section{Fuentes Documentales}

El análisis correspondiente a las características que predomina en el tema seleccionado, llevan a incluir diferentes fuentes documentales encargadas de darle el respectivo apoyo y en ese sentido cumplir con la valoración de los hechos a fin de generar nuevos criterios que sirven de referencia a otros procesos investigativos. Para (CASTRO, 2016) las fuentes documentales incorporadas en la investigación documental o bibliográfica, "representa la suma de materiales sistemáticos que son revisados en forma rigurosa y profunda para llegar a un análisis del fenómeno".(p.41). Por lo tanto, se procedió a cumplir con la realización de una lectura previa determinada para encontrar aquellos 
Mecanismos de prevencion para reducir problemas con el oido causado por la exposición al ruido

aspectos estrechamente vinculados con el tema, con el fin de explicar mediante un desarrollo las respectivas apreciaciones generales de importancia.

\section{Técnicas para la recolección de la información}

La conducción de la investigación para ser realizada en función a las particularidades que determinan a los estudios documentales, tiene como fin el desarrollo de un conjunto de acciones encargadas de llevar a la selección de técnicas estrechamente vinculadas con las características del estudio. En tal sentido, (Bolívar, 2015), refiere, que es "una técnica particular para aportar ayuda a los procedimientos de selección de las ideas primarias y secundarias". (p. 71).

Por ello, se procedió a la utilización del subrayado, resúmenes, fichaje, como parte básica para la revisión y selección de los documentos que presentan el contenido teórico. Es decir, que mediante la aplicación de estas técnicas se pudo llegar a recoger informaciones en cuanto a la revisión bibliográfica de los diversos elementos encargados de orientar el proceso de investigación. Tal como lo expresa, (Bolivar, 2015) "las técnicas documentales proporcionan las herramientas esenciales y determinantes para responder a los objetivos formulados y llegar a resultados efectivos” (p. 58). Es decir, para responder con eficiencia a las necesidades investigativas, se introdujeron como técnica de recolección el método inductivo, que hizo posible llevar a cabo una valoración de los hechos de forma particular para llegar a la explicación desde una visión general.

Asimismo, se emplearon las técnicas de análisis de información para la realización de la investigación que fue ejecutada bajo la dinámica de aplicar diversos elementos encargados de determinar el camino a recorrer por el estudio, según, (Bolívar, 2015) las técnicas de procesamiento de datos en los estudios documentales "son las encargadas de ofrecer al investigador la visión o pasos que debe cumplir durante su ejercicio, cada una de ellas debe estar en correspondencia con el nivel a emplear" (p. 123). Esto indica, que para llevar a cabo el procesamiento de los datos obtenidos una vez aplicado las técnicas seleccionadas, tales como: fichas de resumen, textual, registros descriptivos entre otros, los mismos se deben ajustar al nivel que ha sido seleccionado. 


\section{Resultados.}

Mantener el cuidado adecuado y la protección en el órgano del oído debería ser uno de los puntos más prioritarios de cada ser humano, los procesos industriales casi siempre son ambientes en donde el ruido es muy alto lo que representa ser escenarios perfectos para causar problemas de audición en los trabajadores.

Aproximadamente 30 millones de personas están expuestos al ruido peligroso en sus puestos de trabajo, de los cuales nueve millones más están en riesgo de pérdida de audición inducida por el ruido que generan los equipos industriales. La pérdida de audición es una de las enfermedades más comunes en el ámbito ocupacional y la segunda más auto-informada. El (NIOSH, 2008) en su estudio revela que personas que trabajan en el área de la carpintería 44\% de ellos así como también $48 \%$ fontaneros expresaron tener perdida de la audición, en el área de minería $49 \%$ de los hombres también expresaron tener problemas de audición al llegar a la edad de los 50 años.

Tomando en consideración que cualquier trabajador puede estar en riesgo de ruido inducido y pérdida de audición en el lugar de trabajo, muchas industrias representan una de las mayores causas de exposición a niveles peligrosos de ruido. Entre las áreas industriales con alto número de expuestas está: la agricultura, minería, construcción, empresas de fabricación y servicios públicos, transporte entre otros.

Como se menciona anteriormente el oído está compuesto por tres partes: el oído externo, el oído medio y el oído interno tal como se aprecia en la figura $\mathrm{N}^{\mathrm{o}} 1$. 
Fig. Nor1. Partes del oído

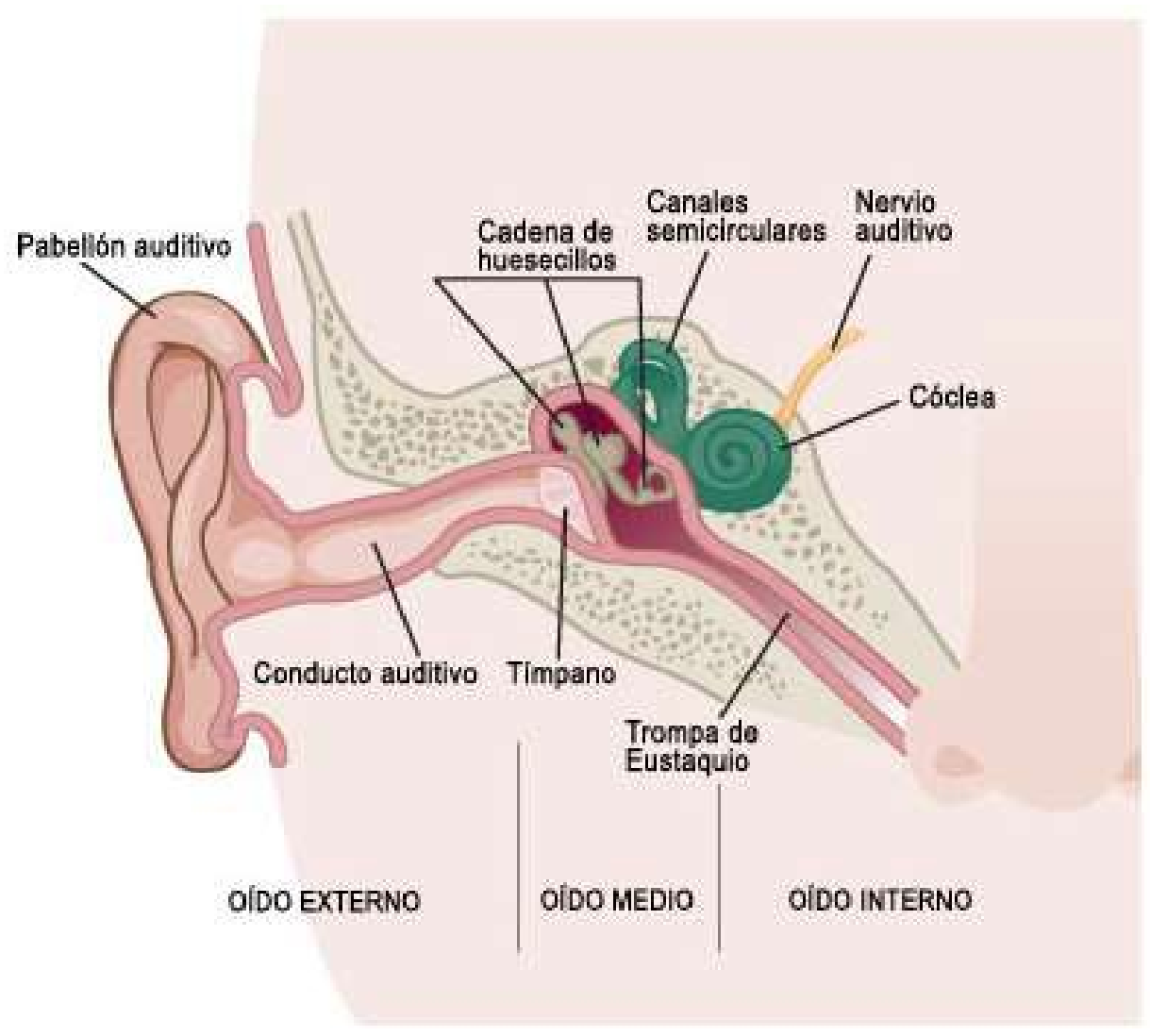

Fuente: (Dowshen, 2011).

El oído externo: Es la parte que resulta visible a un lado de la cabeza, se denomina pabellón auditivo (también conocido como pabellón auricular pabellón de la oreja). Consta de cartílago cubierto de piel. La principal función del pabellón auricular es captar o recibir sonidos y conducirlos hacia el conducto auditivo, que conecta con el oído medio. El pabellón auditivo, que incluye el lóbulo de la oreja, es la parte que se perfora la gente para llevar pendientes.

El oído medio: Es una cavidad llena de aire de aproximadamente el tamaño de un guisante. Transforma las ondas sonoras en vibraciones y las transmite al oído interno. El oído medio está separado del externo por el tímpano o membrana timpánica, una fina lámina de tejido en forma de cono que está fuertemente tensada sobre la luz del conducto auditivo.

El oído interno: Consta de dos órganos diminutos denominados cóclea (o laberinto) y canales semicirculares. La cóclea, en forma de caracol, actúa como una especie de micrófono, 
transformando las vibraciones del oído medio en impulsos nerviosos que viajan hacia el cerebro a lo largo del nervio coclear, también conocido como nervio auditivo.

El Programa de Prevencion Auditiva del Instituto Nacional de Seguridad y Salud Ocupacional se enfoca en reducir la perdida audutiva ocupacional en la industria, las asociaciones comerciales, las organiazaciones, entre otros, a traves de trabajos de investigacion que se realizan en los puestos de trabajo a modo de controlar los ruidos peligrosos y garantizar el uso adecuado de los protectores auditivos sobretodo en lugares en donde el empelado este expuesto a ruido peligroso. (NIOSH, 2017).

Las investigaciones se orienta en desarrollar y evualuar controles para reducir la exposicion a niveles de ruido peligroso por medio de la vigilancia de la audicion ocupacional monitoreando el progreso en la prevencion. El programa promueve las mejores prácticas basadas en la evidencia para disminuir la pérdida de audición relacionada con el trabajo, a traves, del desarrollo de productos de comunicación NIOSH, incluyendo pautas y documentos de criterios, impulsando el uso de los protectores auditivo avanzados con tecnología de prueba de ajuste para asegurar que los trabajadores estén protegidos y buscando identificar, caracterizar y reducir los factores de riesgo asociado con la pérdida de audición inducida por ruido, especialmente en la exposición al ruido intermitente e impulsivo que son los que presenten mayor riesgo.

Las investigaciones han sido realizadas a trabajadores bajo las normas ANSI / ASA S3.44-2016, ya que, esta norma se centra en exposiciones relacionadas con el trabajo y estima el riesgo de pérdida auditiva, el estudio arrojó resultados en la pérdida de audición significativos según la edad del personal que trabaja en el área de minería a continuación se expresa en un grafico lo anteriormente descrito. 
Mecanismos de prevencion para reducir problemas con el oido causado por la exposición al ruido

Grafico $N^{\circ} 1$. Encuesta de Salud Nacional y Examen de Nutrición 2011-2012. Signos vitales: pérdida auditiva inducida por el ruido entre adultos - Estados Unidos

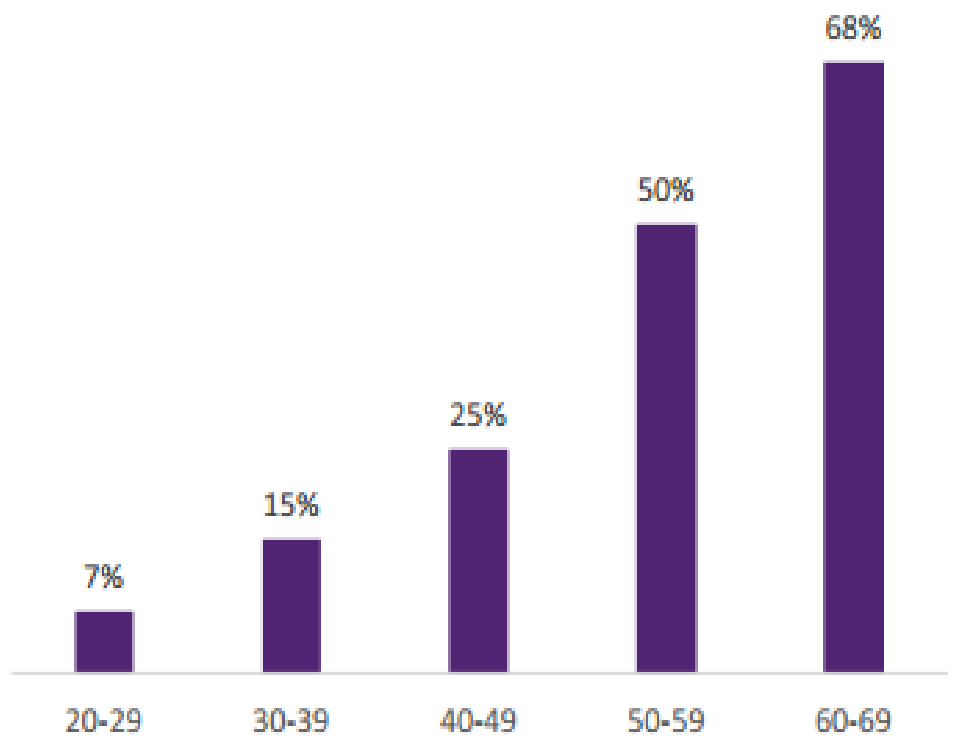

Fuente: (NIOSH, 2017).

En la grafica $\mathrm{N}^{\mathrm{o}} 1$ se observa, como el avance de la perdida de audición en trabajadores a medida que avanzan de edad es bastante notable y que la exposición al ruido causa a largo plazo daños colaterales en éste órgano, por tal motivo se hace énfasis a los mecanismos de prevención necesarios para reducir los riesgos de perdida de audición por exposicion al ruido.

La aplicación de controles de ruido fue realizada a las maquinas mineras continuas con cadenas transportadoras de doble rueda dentadas a continuación se presenta un grafico de lo anteriormente descrito: 
Marco V. Peñaherrera-Alcivar; Marcos A. Bedoya-Romo; Pierina E. Saltos-Montes; Evelyn E. Calderón-López; Marvin D. Veliz-Mero; Marcos A. Campozano-Burgos

Gráfico $N^{2}$. Porcentaje del control de ruido en máquinas mineras continuas con cadena transportadora de doble rueda dentada, 2009-2014

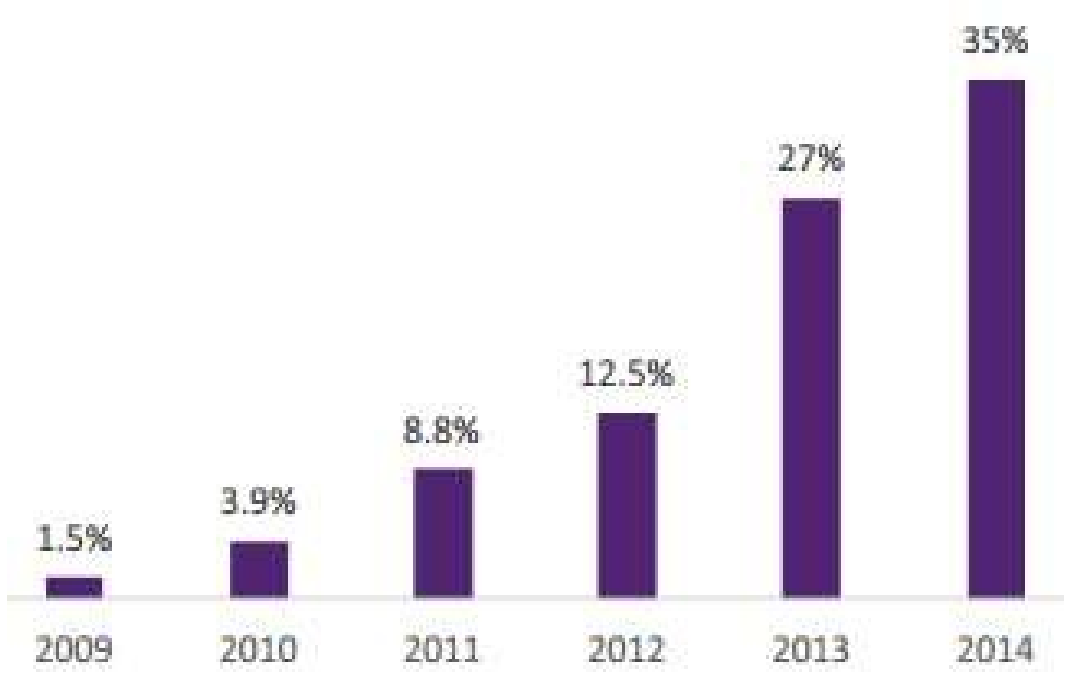

Fuente: (NIOSH, 2017).

En el grafico $\mathrm{N}^{\circ} 2$ se observa como los controles de prevención aplicados desde el 2009 han logrado controlar el ruido en la industria de minería en un 35\% lo que resulta bastante beneficioso para los trabajadores reduciendo el riesgo de pérdida de audición inducida causados por ruidos en sus puestos de trabajo.

Los trabajos que se realizan en la rama de la construcción representan una de las zonas laborales con mayor riesgos de pérdida de audición por ruido, (The Center for Contrucition Research and Training, 2018) en su Programa de Entrenamiento para la Prevención de la Pérdida Auditiva por Ruido de Construcción expone en la tabla $\mathrm{N}^{\circ} 1$ los limites de ruido aprobados por la NIOSH y la OSHA (Administración de Seguridad y Salud Ocupacional).

Tabla $N^{o} 1$. Límites de ruido permitidos en la construcción (dBA)

\begin{tabular}{|c|c|c|}
\hline $\begin{array}{c}\text { Duración por día en } \\
\text { horas }\end{array}$ & NIOSH recomendada & $\begin{array}{c}\text { OSHA Norma de } \\
\text { construcción. }\end{array}$ \\
\hline 8 & 85 & 90 \\
\hline 4 & 88 & 95 \\
\hline 2 & 91 & 100 \\
\hline
\end{tabular}


Mecanismos de prevencion para reducir problemas con el oido causado por la exposición al ruido

\begin{tabular}{|c|c|c|}
\hline 1 & 94 & 105 \\
\hline $1 / 2$ & 97 & 110 \\
\hline $1 / 4$ & 100 & 115 \\
\hline
\end{tabular}

Fuente: (CPWR, 2018).

La tabla muestra que los trabajadores pueden estar expuestos a ruido según cierto período de tiempo considerando que el límite de exposición recomendado (REL) por el NIOSH es de 85 decibelios para un día de 8 horas, mientras que la OSHA expresa que el límite de exposición permitido (PEL) para el ruido es de 90 decibelios para un día de 8 horas. De tal manera se deben utilizar controles administrativos y de ingeniería factibles, y si estos fallan reducir los niveles de sonido al PEL.

Por otra parte, los trabajadores deben usar dispositivos de protección auditiva y recibir capacitación sobre cómo usarlos adecuadamente, de acuerdo al PEL si es el límite es el legal promediado en un día laboral de 8 horas para exposición al ruido del lugar de trabajo, a menos que se usen controles o protección especial. La NIOSH ha recomendado a OSHA que actualice su PEL a un REL de 85 decibelios durante 8 horas al día declarando que las exposiciones en y por encima de este nivel se consideran peligrosas.

Los estudios e investigaciones que se han realizado tanto en casos específicos como generales, resultan un tema bastante importante, considerando indispensable adaptar mecanismos de prevención que minimicen los riesgos de presentar problemas con el oído debido a la exposición recurrente al ruido. El autor (Prevencion, 2017) expone algunos mecanismos que se deben tomar en cuenta:

- Establecer las mejoras y correcciones necesarias en los diferentes puestos de trabajo, elaborando el correspondiente programa de prevención.

- Realizar un estudio de cada una de las fuentes de ruido presentes en la industria.

- Establecer un programa de control cuyo objetivo sea conseguir un nivel de ruido lo más bajo posible en los puestos de trabajo.

- Utilizar los métodos de protección que aíslen y protejan al trabajador del ruido ambiental.

En las empresas debe exigir medidas de prevención tales como: 
- Utilizar los métodos de protección que aíslen y protejan al trabajador del ruido ambiental.

- Eliminar el origen de los riesgos derivados a la exposición al ruido o reducirlos al nivel más bajo posible mediante la adopción de las medidas técnicas generales.

- Asegurar que la exposición de ningún trabajador sea superior al valor límite de exposición y adoptar las medidas necesarias en caso de que se superen.

- Realizar una evaluación basada en la medición de niveles de ruido a los que los trabajadores están expuestos.

- Proporcionar los equipos de protección individual convenientes y velar por un uso adecuado.

- Garantizar la formación e información adecuada.

- Garantizar una vigilancia adecuada y específica de la salud.

\section{Discusión y conclusiones.}

Siempre se está expuesto a ruidos, bien sean en la cotidianidad de la vida o en cualquier puesto de trabajo, mediante el desarrollo del proceso investigativo, se hizo énfasis en los riesgos de pérdida de audición que se tienen en los lugares de trabajos por no tomar los mecanismos de prevención adecuados que logren evitar este daño a los oídos.

Un gran número de personas se encuentran diariamente expuestas a ruidos peligros en los puestos de trabajo trayendo como consecuencia la pérdida parcial o total de la audición con el pasar de los años. En la investigación se observó que trabajadores del área de la carpintería, fontaneros, mineros, entre otros, afirmaron presentar pérdida de audición con los años debido a la exposición que tuvieron al ruido por largo periodo de tiempo.

Sin embargo, existen programas como el Programa de Prevencion Auditiva del Instituto Nacional de Seguridad y Salud Ocupacional (NIOSH) que se encargan de realizar las evaluaciones y controles pertinentes en las empresas y organizaciones a fin de mantener los cuidados adecuados en el area y garantizar la audición de los trabajadores. 
Mecanismos de prevencion para reducir problemas con el oido causado por la exposición al ruido

En la investigacion se expresan estudios realizados a trabajadores en el área de minería donde se observó, que aquellos trabajadores de mayor edad presentaron pérdida de audicion por le tiempo de exposición al ruido peligroso, tambien se evaluaron casos de trabajadores de la construcción en donde se observó que el REL es de 85 decibelios para un tiempo de 8 horas laborales mientras que el PEL es de 90 decibelios, cifra que se ha llevado a discusion debido a que el NIOSH recomienda que la OSHA debería ajustar su limite a 85 decibelios considerando que una cifra entre o mayor a 85 produce daños significativos en los órganos de los oidos.

Finalmente se concluye, que las organizaciones y empresas en general deben tener un estricto cuidado de la audición de sus trabajadores, promoviendo jornadas de investigación y controles de seguridad destinados al chequeo de los equipos industriales y exigiendo el uso correcto y necesario de los equipos de protección de seguridad en los empleados que se encuentran expuestos a ruidos peligrosos aplicando, de ser posible, sanciones a aquellos trabajadores que no se coloquen sus equipos y asegurándoles un chequeo médico que garantice la salud del trabajador y logre prevenir los riesgos de pérdida de audición inducida.

\section{Bibliografia.}

Arandia, R., \& Ayala, M. (2010). RECIEN NACIDO “PREMATURO TARDÍO” FRENTE A LOS RIESGOS QUE DEBEN TENERSE ENCUENTA. Gaceta Médica Boliviana (33), N2, 6469.

Bolívar, J. (2015). Investigación Documental. México. Pax.

Castro, J. (2016). Técnicas Documentales. México. Limusa.

CPWR. (18 de Febrero de 2018). The Center for Contrucition Research and Training. Recuperado el 1 de MAyo de 2019 , de https://www.cpwr.com/sites/default/files/Construction\%20Noise $\% 20 \% 26 \% 20$ Hearing\%20L oss $\% 20-\% 20$ Instructor\%20Guide $\% 20$ \%2030\%20Minute\%20Program\%202.27.2018\%20\%283\%29.pdf

Davila, A. (2015). Diccionario de Términos Científicos. . Caracas: Editorial Oasis.

Díaz, V. (2005). Lactancia materna: evaluación nutricional en el recién nacido. Revista Cubana de Pediatría, vol 77. N2, http://scielo.sld.cu/scielo.php?script=sci_arttext\&pid=S003475312005000200005.

Dowshen, S. (2011). Wake Forest. Recuperado el 31 de Abril de 2019, de https://www.brennerchildrens.org/KidsHealth/Parents/Cerebral-Palsy-Center/En-espanol/Eloido.htm 
Marco V. Peñaherrera-Alcivar; Marcos A. Bedoya-Romo; Pierina E. Saltos-Montes; Evelyn E. Calderón-López; Marvin D. Veliz-Mero; Marcos A. Campozano-Burgos

FREMAP. (2018). Universidad Complutence Madrid. Recuperado el 29 de Abril de 2019, de https://www.ucm.es/data/cont/docs/3-2013-02-19-12-

\%20ME.TRI.018\%20exposici\%C3\%B3n\%20al\%20ruido.pdf

Lizarazo-Medina, J. P., Díaz, O., M., J., \& Ariza Riaño, N. E. (2012). Programa madre canguro: una alternativa sencilla y costo eficaz para la protección de los recién nacidos prematuros o con bajo peso al nacer. Revista de Salud Pública, 32-45.

Martínez Contreras, A., Soria Rodríguez, C., Prince Vélez, R., Clark Ordoñez, I., \& Medina Ramírez, M. (2008). 398GinecoloGíay obstetriciade MéxicoPreeclampsia: principal factor de riesgo materno para bajo peso del recién nacido pretérmino. GinecoloGíay obstetriciade México, 398-403.

Mata, M., Salazar, M., \& Herrera, L. (2009). Cuidado enfermero en el recién nacido prematuro. Revista Enfermería Instituto Mexicano de Seguro Social, 45-54.

NIOSH. (2008). National Institute for Occupational Safety and Health Work-related hearing loss. Recuperado el 29 de Abril de 2019, de at: http://www.cdc.gov/niosh/01-103.html. Accessed

Prevencion, S. d. (2017). MAZ, Sociedad de Prevencion . Recuperado el 1 de Mayo de 2019, de https://www.spmas.es/media/1140/recomendaciones_ruido.pdf

Sarmiento Portal, Y., Crespo Campos, A., Portal Miranda, M., Morales Delgado, I., \& Piloña Ruíz, S. (2009). Análisis de la morbilidad y mortalidad en recién nacidos con peso inferior a 1500 g Revista Cubana de Pediatría, http://scielo.sld.cu/scielo.php?script=sci_arttext\&pid=S0034-75312009000400002.

Velázquez Quintana, N. I., Masud Yunes Zárraga, J. L., \& Ávila Reyes, R. (2004). Recién nacidos con bajo peso; causas, problemas y perspectivas a futuro. Boletín médico del Hospital Infantil de México. Vol 1., 73-86. 